\title{
Percepción de mejora en el aprendizaje de una segunda lengua
}

\author{
Karen McMullin. Trent University \\ Recepción: 10 de febrero de 2016 | Revisión: 20 de febrero de 2016 | Aceptado: 22 febrero de 2016 \\ Correspondencia: karenmcmullin@trentu.ca \\ Citar: McMullin, K. (2016). Percepción de mejora en el aprendizaje de una segunda lengua. ReiDoCrea, 5, $22-32$.
}

Resumen: Collage es una metodología de enseñanza de las lenguas que se basa en la lectura de textos auténticos siguiendo un procedimiento innovador que es a la vez específico y flexible. El objetivo de este estudio es analizar la percepción de mejora en el aprendizaje de una segunda lengua, a través del método Collage. Participaron 15 estudiantes universitarios de un curso de Lengua Española completando un cuestionario sobre su percepción de mejora en diferentes aspectos del aprendizaje de la lengua. Los resultados fueron favorables, mostrando una valoración positiva de la experiencia, que llevan a la conclusión que el método Collage se percibe como eficaz en la enseñanza del español.

Palabras clave: Enseñanza de una segunda lengua | Lectura

Perception of Improvement in Learning a Second Language

Abstract: Collage is a language teaching methodology based on reading authentic texts following an innovative procedure that is both specific and flexible. The aim of this study is to analyze the perception of improvement in learning a second language using the Collage method. Fifteen university students in a Spanish Language course participated, completing a questionnaire on their perception of improvement in different aspects of language learning. The results were favorable, showing a positive assessment of the experience, which leads to the conclusion that Collage is perceived as an effective method of teaching Spanish.

Keywords: Second Language Instruction | Reading

El aprendizaje de las lenguas es un proceso complejo en el que intervienen factores internos como la motivación, aptitud, estilo de aprendizaje y conocimientos previos del alumnado, y factores externos como el contexto y la propia distribución del aprendizaje. La mayoría de los cursos de lengua impartido a nivel universitario se basan en el enfoque comunicativo con un currículum organizado alrededor de un programa nocional-funcional. Aunque se enseñan las cuatro destrezas, el énfasis a menudo recae en la práctica oral de vocabulario situacional. Ante este escenario, el profesorado de lenguas se enfrenta con el reto de conseguir que su alumnado se exprese correctamente, con confianza y buena pronunciación. El alumnado, por su parte, se encuentra en la obligación de participar en esta práctica oral sin que se tenga en cuenta su estilo de aprendizaje, personalidad, motivación, confianza, comodidad o intereses. Sin embargo existen enfoques que se centran más en la comprensión lectora, toman el texto escrito como punto de partida con el análisis de estructuras gramaticales no graduadas, permitiendo que el alumnado manipule y practique la lengua, acumulando una base léxica y gramatical a la vez que practica la expresión oral contextualizada en un entorno cooperativo. La presentación, práctica y aprendizaje de vocabulario en contexto es un aspecto importante del método Collage (Möller 2001; McMullin, 2012, 2013) en el que se anima al alumnado a usar el vocabulario aprendido dentro del contexto de los textos y material de apoyo. De esta manera, se consolida la asimilación de palabras y frases, facilitando la retención y marcando el progreso de los estudiantes en el proceso de aprendizaje. Por lo tanto, este método, al basarse en la lectura de textos escritos, ayuda a aumentar el léxico y la confianza del alumnado para que pueda participar activamente y sin miedo en la expresión oral de temas relevantes y significativos. También el procedimiento flexible de este método permite la acomodación de los diferentes niveles, estilos de aprendizaje y necesidades del alumnado ajustando la programación, formación de grupos y presentación de los textos. Asimismo, se tienen en cuenta los intereses de los estudiantes al elegir los textos para un grupo particular. La flexibilidad del método también permite la inclusión de diferentes modalidades de presentación o tipos de 
textos y material de apoyo, teniendo en cuenta los avances recientes en el área de la TIC y su creciente conexión con la educación y el aprendizaje (Garcia, 2014; Marín, Vázquez y McMullin, 2014; Sampedro y McMullin, 2015). Así como el uso de la metodología interpretativa, concretamente de investigación-acción, con la idea de mejorar el nivel de implicación tanto del profesorado como del alumnado, contextualizando el conocimiento, potenciando el aprendizaje participativo y colaborativo, la acción-reflexión y la investigación en la adquisición y desarrollo de competencias (Madrid, 1998; Garcia, 2012, 2016; Fiorini y Garcia, 2013).

Las características de participación, interacción, reflexión y construcción de conocimientos de esta metodología reflejan las características del método de enseñanza Collage que anima a estudiantes y profesorado a reflexionar sobre estudio de lenguas, las posibilidades laborales y sociales que éstas posibilitan, el nivel que creen que deberían y podrían alcanzar además del uso de su experiencia en el su aprendizaje (Möller, 2001; McMullin, 2013). Por lo tanto, es importante que el alumnado sepa qué se espera de él y qué objetivos debe alcanzar, para que sea responsable de su propio aprendizaje (Dickinson, 1987; McMullin, 2011; McMullin y Garcia, 2015; Valencia y Garcia, 2013). Así que el método se pone en práctica a través de un procedimiento de trabajo específico y a la vez flexible basado en líneas generales, no únicas, de actuación en la que se mantiene una estructura procedimental de presentación, lectura y análisis de los textos (Möller, 2001; McMullin, 2013).

En un estudio realizado en Trent University se realizó una adaptación del método Collage al curso Continuing Spanish. El alumnado tenía dos clases semanales de seminario dedicadas a la presentación, lectura y análisis de los textos que iban a usar. También disponían de una hora de conversación semanal en la que se discutían los temas de los textos y otros relacionados; posteriormente durante otra hora semanal de laboratorio continuaban con el análisis de los textos, buscaban información en Internet para aclarar el contenido de los textos, o hacían ejercicios adicionales. En este último momento se introducían textos complementarios de diferentes modalidades como vídeos, canciones y páginas de redes sociales, para seguir desarrollando la comprensión auditiva además de la lectora y proporcionar apoyo visual. En este proceso de adaptación es fundamental la autonomía del alumnado y el trabajo cooperativo, para adquirir la competencia comunicativa y la competencia intercultural a partir de la comprensión lectora de los texto, la reflexión sobre su proceso de aprendizaje y la relación de los conocimientos previos con los conceptos nuevos; también es clave la adquisición y uso de las habilidades necesarias para entender los textos que tenían que leer y analizar de forma autónoma fuera del aula, potenciando la confianza en sí mismos, en sus conocimientos y su capacidad de comprender el sentido general de los textos. Otro aspecto relevante es el desarrollo y uso de la competencia intercultural que ayuda a comprender los textos de forma significativa, facilitando el aprendizaje de la lengua meta. Por lo tanto, el método Collage se centra en el desarrollo de la comprensión lectora, formando una base firme sobre la cual construir el conocimiento de la lengua meta y desarrollar las destrezas necesarias para su dominio. Finalmente los resultados de implementar el método Collage en un grupo fueron positivos; el análisis estadístico de la comparación de las medias de la evaluaciones obtenidas de los participantes del grupo al que se implementó el método fue significativa $(M=83,00, S E=2,855, t(67)=-2,538, p=0,013, r=0,3)$ respecto a las medias de las evaluaciones de los participantes de otros grupos con los que se usaba otra metodología diferente y habitual $(M=74,13, S E=1,66)$. A pesar de esta evidencia, en ese estudio se concluye con la necesidad de comprobar la percepción de los/as participantes. (McMullin, 2016) 
Por lo tanto, el objetivo de este estudio es analizar la percepción de mejora en el aprendizaje de una segunda lengua, a través del método Collage.

\section{Método}

\section{Participantes}

La muestra participante en este estudio estaba formada por el alumnado de la asignatura Continuing Spanish que estudiaban la lengua española como tercera lengua. La muestra estaba constituida por 15 estudiantes $(n=15)$, tres hombres y doce mujeres, entre 18 y 22 años de edad $(S D=2.2)$. Los/as participantes estudiaban diferentes titulaciones: Administración de Empresas, Ciencias Ambientales, Antropología, Desarrollo Internacional, Psicología, Ciencias Políticas, Ciencias Forenses, Filología Francesa, Filología Inglesa, Románicas o Hispánicas. Los objetivos y necesidades de los/las estudiantes también eran variados, destacando: mejorar la comprensión lectora y dando considerable importancia también a la comprensión y producción oral para tener la opción de estudiar, investigar o trabajar en países hispanoparlantes. Por lo tanto, la mayoría estudiaban español para acceder a estudios de postgrado o para aumentar sus oportunidades en el mercado laboral o para trabajar en países hispanoparlantes o para complementar su formación en lenguas. Por lo tanto, necesitaban ser capaces de acceder a textos escritos y aumentar el vocabulario en áreas relacionadas con sus futuras profesiones. Aunque este grupo no tenía una meta laboral común, todos reconocían la importancia de las lenguas y particularmente de la lengua española. Esta información se obtuvo a través de un cuestionario preliminar con cinco dimensiones: conocimiento de lenguas, experiencia y actitud, estilo de aprendizaje, áreas de estudio e intereses, y expectativas. El cuestionario fue distribuido en clase, completado y entregado posteriormente (anexo-1).

\section{Instrumentos}

Se utilizó una escala de percepción, tipo Likert, con seis dimensiones: a) contenido de textos y preguntas, b) grado de mejora, c) método, d) conocimientos del español, e) actitud, f) curso. La meta de estas dimensiones era determinar la opinión y nivel de satisfacción de los participantes. (anexo-2)

\section{Procedimiento}

La escala fue cumplimentada por los/as participantes después de finalizar el curso académico.

\section{Análisis de datos}

Los datos obtenidos del instrumento fueron analizados cualitativamente, atendiendo la frecuencia.

\section{Resultados}

Los resultados muestran la satisfacción general del alumnado con el método, el curso y el contenido de los textos y las preguntas. En la dimensión de contenido el $60 \%$ $(n=9)$ del alumnado estaba de acuerdo que el contenido de los textos era apropiado para su nivel de conocimientos de la lengua; el $80 \%(n=12)$ estaba de acuerdo que los temas y contenido de los textos eran interesantes; el $73 \%(n=11)$ creía que el contenido era útil; el $67 \%(n=10)$ contestó que las preguntas eran fáciles de 
comprender. El 53\% $(n=8)$ estaba de acuerdo que las preguntas le ayudó a comprender los textos y el $40 \%$ no estaba seguro. Sólo el $20 \%(n=3)$ creía que el número de textos era apropiado y el $53 \%$ no estaba de acuerdo. El $47 \%(n=7)$ creía que la extensión de los textos era adecuado y el $27 \%$ no estaba de acuerdo. Los datos de esta dimensión se exponen en la tabla 5 y el gráfico 5 .

\begin{tabular}{|c|c|c|c|}
\hline \multicolumn{4}{|c|}{ Tabla 5. Contenido } \\
\hline Questions & Agree/Strongly agree & Disagree/Strongly disagree & Not sure \\
\hline The content of the texts was appropriate for my language level & 9 & 2 & 4 \\
\hline The themes and content of the texts were interesting & 12 & 1 & 2 \\
\hline The content of the texts was useful & 11 & 1 & 3 \\
\hline The number of texts was appropriate & 3 & 8 & 4 \\
\hline The texts were a good length & 7 & 4 & 4 \\
\hline The questions were easy to understand & 10 & 1 & 4 \\
\hline The questions helped me to understand the text & 8 & 1 & 6 \\
\hline
\end{tabular}

Gráfico 5. Contenido
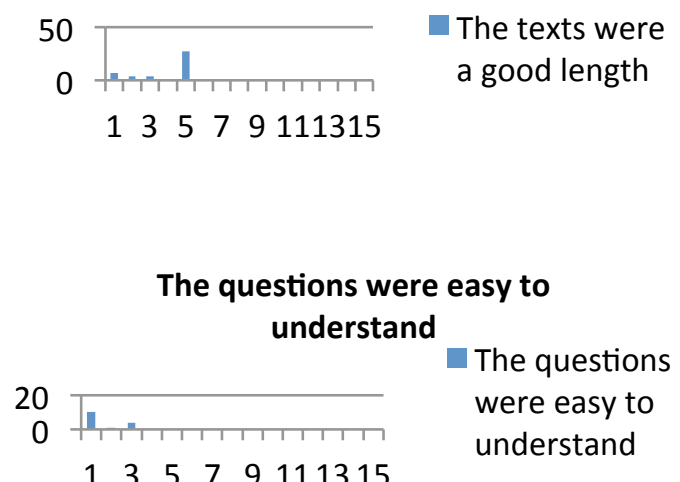

$\begin{array}{lllllll}1 & 3 & 5 & 7 & 9 & 1113 & 15\end{array}$

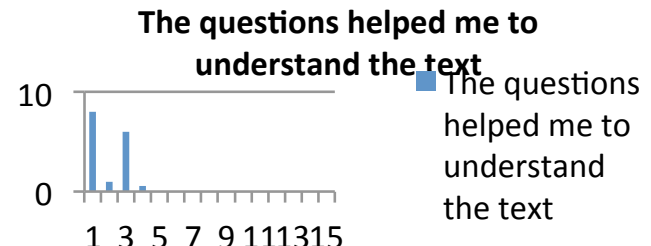

The content of the texts was appropriate for my language level

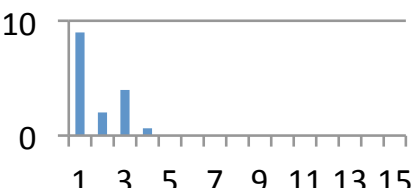

The content of the texts was appropriate for my language level

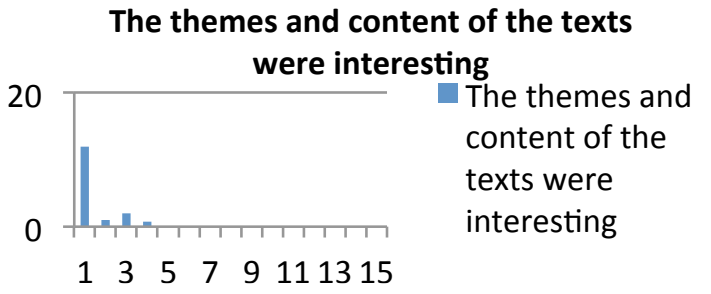

The content of the texts was useful

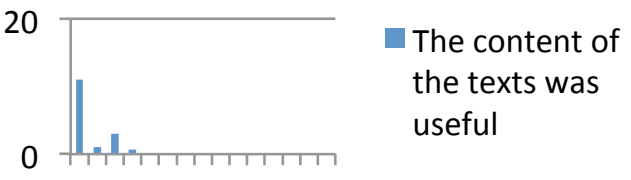

13579111315

The number of texts was appropriate

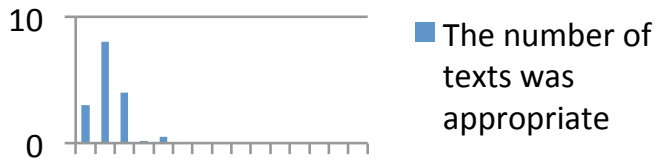

1335770111315

En la dimensión de mejora los participantes dieron una valoración muy positiva. El $93 \% \quad(n=14)$ del alumnado opinaban que su conocimiento de contenido había mejorado; el $80 \%(n=12)$ creían que su nivel de confianza había mejorado; el $93 \%$ $(n=14)$ contestaron que había aumentado su vocabulario y el $93 \%$ también creían que había mejorado su comprensión. Sólo hubo una valoración negativa en el ítem de la pronunciación donde el $53 \% \quad(n=8)$ contestaron que no había mejorado su pronunciación y sólo el $47 \%(n=7)$ contestaron que había mejorado. Los datos de esta dimensión se exponen en la tabla 6 y el gráfico 6. 


\begin{tabular}{|c|c|c|c|}
\hline \multicolumn{4}{|c|}{ Tabla 6. Grado de mejora } \\
\hline Questions & Agree/Strongly agree & Disagree/Strongly disagree & Not sure \\
\hline I feel that there was improvement in my knowledge of content & 14 & 0 & 1 \\
\hline I feel that there was improvement in my pronunciation & 7 & 0 & 8 \\
\hline My level of confidence with the language improved & 12 & 1 & 2 \\
\hline I feel that I have increased my vocabulary & 14 & 0 & 1 \\
\hline My comprehension has improved & 14 & 0 & 1 \\
\hline
\end{tabular}

Gráfico 6. Grado de mejora

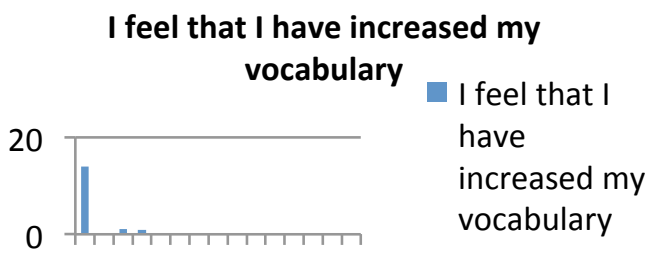

13579111315

My comprehension has improved

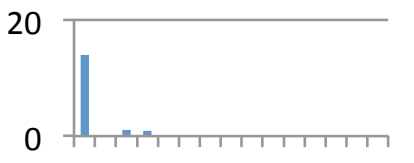

1355799111315

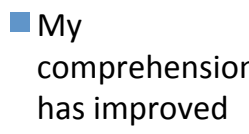

has improved
I feel that there was improvement in my knowledge of content

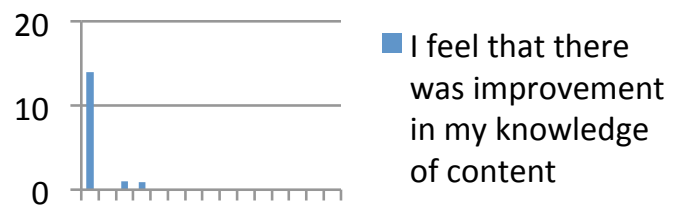

13579111315

I feel that there was improvement in my pronunciation

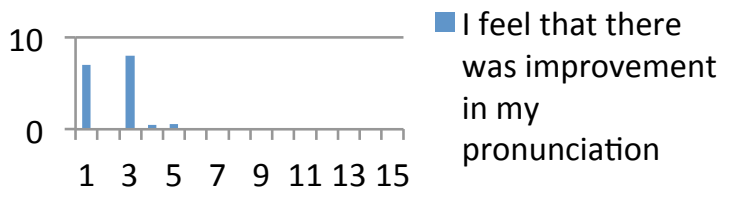

My level of confidence with the language improved

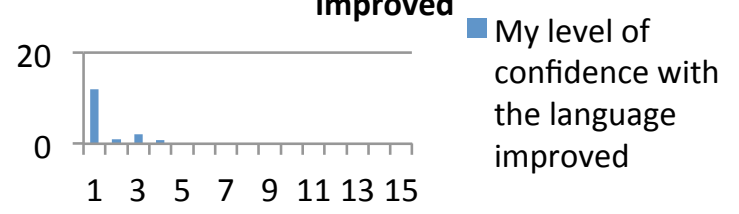

En la dimensión de método aunque sólo el $47 \% \quad(n=7)$ contestaron que se sentían cómodos usando este método, al $67 \%(n=10)$ de los participantes les gustó trabajar de forma autónoma y al $87 \%(n=13)$ les gustó cooperar con los compañeros y con la profesora; el $73 \%(n=11)$ opinaban que su conocimiento de otras lenguas les ayudó a comprender la lengua meta; al $87 \%(n=13)$ les gustó leer sobre personas y lugares de habla española y el $93 \%(n=14)$ contestaron que aprendieron sobre la cultura de estos países. El $60 \%(n=9)$ contestaron que participaron activamente en el proceso de aprendizaje; el $93 \%(n=14)$ afirmaron haber aprendido mucho sobre la lengua española. Sólo el 53\% $(n=8)$ contestaron que aprendieron cuándo y cómo usar recursos para encontrar información. Los datos de esta dimensión se exponen en la tabla 7 y el gráfico 7 .

\begin{tabular}{|lccc|}
\hline \multicolumn{4}{|c|}{ Tabla 7. Método } \\
\hline Questions & Agree/Strongly agree & Disagree/Strongly disagree Not sure \\
\hline I felt comfortable using this method & 7 & 3 & 5 \\
I enjoyed working independently to understand the texts & 10 & 4 & 1 \\
I enjoyed working cooperatively with my classmates and the & 13 & 0 & 2 \\
instructor & & 0 & 4 \\
My knowledge of other languages helped me to understand Spanish & 11 & 2 & 5 \\
I learned when and how to use resources to find information & 8 & 0 & 2 \\
I enjoyed reading about people and places in Spanish & 13 & 0 & 1 \\
I learned about the culture of Spanish-speaking countries & 14 & 0 & 6 \\
I participated actively in the learning process & 9 & 1 & 0 \\
I learned a lot about the Spanish language & 14 & & \\
\hline
\end{tabular}


Gráfico 7. Método

I enjoyed reading about people and places in Spanish

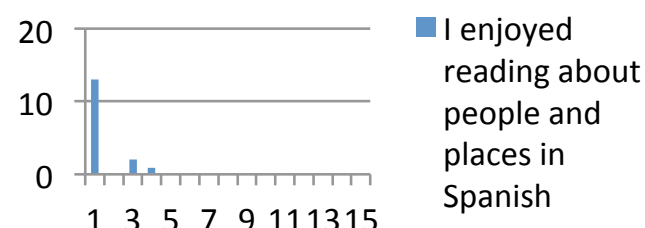

I learned about the culture of Spanish-speaking countries

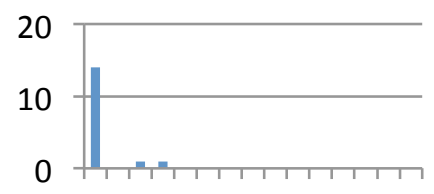

$\begin{array}{llllllll}1 & 3 & 5 & 7 & 9 & 11 & 13 & 15\end{array}$

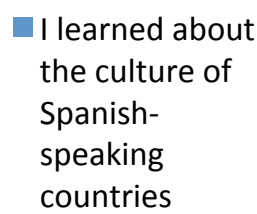

I participated actively in the learning process

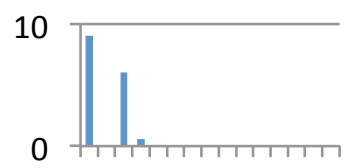

I participated
actively in the
learning process

13579111315

I learned a lot about the Spanish language

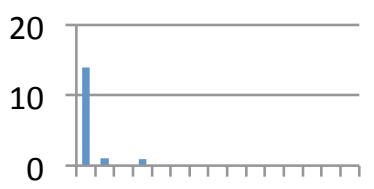

133579111315

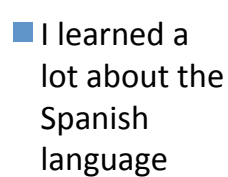

language
I felt comfortable using this

method I felt

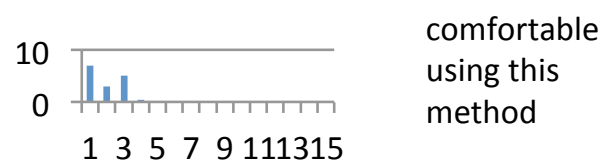

13579111315

I enjoyed working independently to understand the texts

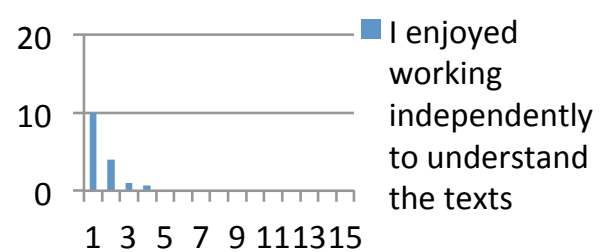

I enjoyed working cooperatively with my classmates and the

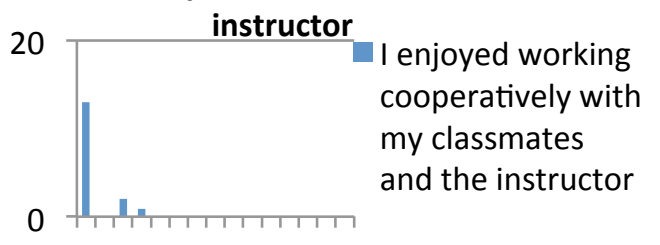

1355799111315

My knowledge of other languages helped me to understand Spanish

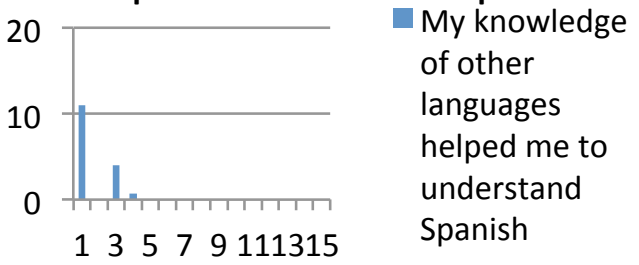

I learned when and how to use resources to find information

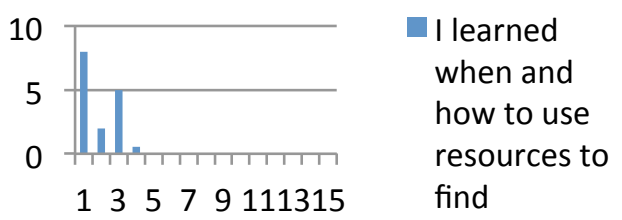

En la dimensión de curso sólo el $47 \%(n=7)$ de los participantes contestaron que les gustó el hecho de que se centraba en la lectura; el $20 \%(n=3)$ no estaba de acuerdo y el $34 \%(n=5)$ no estaban seguros; y el $80 \%(n=12)$ estaban de acuerdo en que preferían aprender más lengua oral; el $73 \%(n=11)$ opinaban que las actividades del laboratorio y de conversación complementaban las otras clases; el $60 \% \quad(n=9)$ contestaron que se sienten motivados para seguir aprendiendo español, el $40 \%$ no estaban seguros y al $53 \%(n=8)$ les gustaría aprender más con este método. Los datos de esta dimensión se exponen en la tabla 8 y el gráfico 8. 


\begin{tabular}{|c|c|c|c|}
\hline \multicolumn{4}{|c|}{ Tabla 8. Curso } \\
\hline Questions & $\begin{array}{l}\text { Agree/Strongly } \\
\text { agree }\end{array}$ & $\begin{array}{l}\text { Disagree/Strongly } \\
\text { disagree }\end{array}$ & $\begin{array}{l}\text { Not } \\
\text { sure }\end{array}$ \\
\hline I liked the fact that the focus was on reading & 7 & 3 & 5 \\
\hline $\begin{array}{l}\text { The activities of the labs and conversations complemented the } \\
\text { seminars }\end{array}$ & 11 & 0 & 4 \\
\hline I would prefer to learn more oral Spanish & 12 & 1 & 2 \\
\hline I feel motivated to continue to learn Spanish & 9 & 0 & 6 \\
\hline I would like to learn more Spanish using this method & 8 & 3 & 4 \\
\hline
\end{tabular}

I liked the fact that the focus was on reading

Gráfico 8. Curso

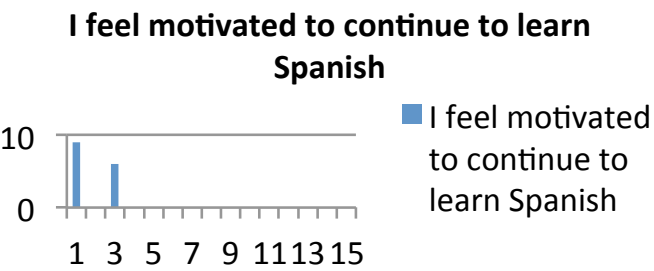

I would like to learn more Spanish using this method

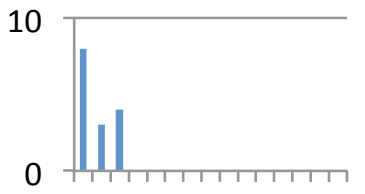

135579111315

\section{- I would like to learn more Spanish using this method}

13579111315

I liked the fact that the focus was on reading

The activities of the labs and conversation complemented the seminars

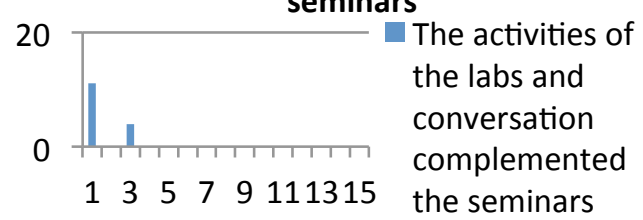

I would prefer to learn more oral Spanish

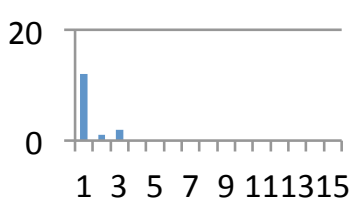

- I would prefer to learn more oral Spanish

\section{Discusión}

Aunque la muestra del grupo de participantes es pequeña los resultados del análisis cualitativo complementan los resultados cuantitativos obtenidos en el estudio experimental realizado simultáneamente durante este estudio (McMullin, 2016). Este estudio evidencia un grado significativo de percepción positiva de aprender la lengua, especialmente en la dimensión de grado de mejora, en las áreas de conocimientos de contenido y de vocabulario y de comprensión, y también en la dimensión de método en los ítems de aprendizaje de la cultura y de la lengua. También destaca la valoración positiva por parte de la mayoría de los/as participantes en el área del trabajo cooperativo y de la lectura de textos sobre personas y lugares de habla española. Pero también es necesario señalar la valoración negativa respecto al número de textos $y$, en menor grado, la extensión de los mismos, por lo que se puede concluir que el número de textos fue excesivo y que algunos fueron demasiado largos. Teniendo en cuenta esta información, se pudo ajustar el número y la extensión de los textos con grupos posteriores con el fin de mejorar los resultados, la valoración y la percepción por parte del alumnado con el método y con el proceso de aprendizaje. También los resultados dieron lugar a explicar la importancia del trabajo autónomo como paso en el procedimiento del método y en el proceso de aprendizaje, y como clave en la toma de responsabilidad del aprendizaje. También los resultados dieron lugar a que se 
modificaran algunas preguntas y se ofreciera aclaración oral en el momento de presentar los textos y las preguntas. Además los resultados mostraron la necesitad de dedicar más tiempo a la explicación y práctica de cuándo y cómo usar los recursos para encontrar información y a la práctica y mejora de la pronunciación. Todo esto en respuesta a la baja valoración en esas áreas y con el fin de mejorar la experiencia y el aprendizaje de la lengua. La mayoría de los/as participantes contestaron que no les gustaba que se centrara en la lectura y que habrían preferido aprender más lengua oral, sin embargo creían que habían mejorado en áreas que eran clave en el aprendizaje de las lenguas. Reduciendo el número de textos y aumentando las actividades relacionadas con la expresión oral, se pudo responder a esta preferencia a la vez que se mantiene la base en la comprensión lectora.

El énfasis en la lectura y análisis de textos auténticos del método Collage es diferente de la metodología a lo que están acostumbrados la mayoría de los estudiantes y abordar el aprendizaje de las lenguas de esta manera les puede resultar incómodo por el temor a lo desconocido, requiriendo un período de reajuste mientras se acostumbran a este procedimiento. Sin embargo, podemos afirmar que tras practicar la comprensión lectora y el análisis de estructuras gramaticales no graduadas en un entorno cooperativo, el alumnado aumenta el léxico y la confianza, facilitando la participación activa y sin miedo en la expresión oral de temas relevantes y significativos motivados por el interés en los temas de los textos. La flexibilidad del procedimiento permite que trabajen a su ritmo y profundicen más o menos según sus conocimientos previos e intereses mientras se les anima a todos a aprender lo máximo de cada texto.

Después de realizar este estudio es evidente que el método Collage aborda, con eficacia, el problema de la heterogeneidad del grupo en cuanto a diferencias de nivel de dominio y experiencia con la lengua meta, permitiendo que el alumnado trabaje a su propio ritmo, que forme grupos de trabajo y contribuya al grupo según sus conocimientos, experiencias e intereses. Al basarse en una progresión textual con diferentes tipos de textos, les proporciona al alumnado un input auténtico y relevante basado en sus estudios e intereses, lo cual le motiva a usar sus conocimientos previos para comprender el contenido y aumentar su conocimiento y uso de la lengua meta. Estos resultados, junto al estudio experimental (McMullin, 2016) indican que el uso del método Collage se percibe como eficaz para la enseñanza del español como tercera lengua.

Por tratarse de una muestra pequeña para el grupo experimental, sería necesario seguir implementando el método con otros grupos para confirmar que es eficiente satisfactorio. También sería interesante explorar su uso con otros grupos en diferentes contextos, incluyendo estudios de postgrado y educación continua.

Referencias

Diaz-Marin, V., Vazquez-Martinez, A., \& McMullin, K. (2014). First steps towards a university social network on personal learning environments. The International Review of Research in Open and Distance Learning, 15(3), 93-119.

Dickinson, L. (1987). Self-instruction in Language Learning. Cambridge: Cambridge University Press.

Fiorini, M. y Garcia-Ramirez, JM. (2013). Cap. 5: Técnicas de grupo y creatividad aplicadas en el ámbito universitario,117-147. En M.D. Villena Martínez y A. Muñoz García (2013). Recursos para la tutoría en el aula universitaria. Granada: Editorial Universidad de Granada.

Garcia-Ramirez, JM. (2014). Selección de indicadores para la evaluación de la excelencia docente en la Universidad de Trent (Canadá). Granada: Universidad de Granada. 
Garcia-Ramirez, JM. (2016). Predictibilidad y creatividad narrativa. ulu, 1, 1-6.

Madrid, D. (1998). Guía para la investigación en el aula de idiomas. Granada: Grupo Editorial Universitario.

McMullin, K. (2011). Aprendizaje cooperativo: Programa de reforzamiento de la lengua española: ¡10 minutos!. Andaluciaeduca, 73, 51-52.

McMullin, K. (2012). Collage: Una orientación metodológica innovadora en la enseñanza de una tercera lengua. Andaluciaeduca, 75, 51-54.

McMullin, K. (2013). Collage: Una metodología de enseñanza de una tercera lengua. redELE: revista electrónica de didáctica del Español Lengua Extranjera, 25, 1-25.

McMullin, K. (2016). Cooperation and Autonomy in Language Learning: An Application of the Collage Method. Porta Linguarum, 25, 93-103.

McMullin, K., \& Garcia-Ramirez, JM. (2015). A Language Reinforcement Program: ¡10 minutos!. ReiDoCrea, $4,28-34$.

Möller Runge, Julia. (2001). Siglo XXI: ¿Innovamos? La enseñanza de una segunda lengua extranjera. España: Editorial Alhulia, S.L.

Sampedro Requena, B. y McMullin, K. (2015). Videojuegos para la inclusión educativa. Digital Education Review, 27, 122-137

Valencia-Garcia, N. y Garcia-Ramirez, JM. (2013). A través de la lectura. ReiDoCrea, 3, 273-284. 


\section{Anexo 1 | PRELIMINARY STUDENT QUESTIONNAIRE}

Name

Age/Year

Thank you for completing this questionnaire. In order to help you get the most out of this part of the course and to better understand the results, I need to have an idea of your knowledge of and experience with languages, your attitude, learning style and your interests.

Please take some time to answer the questions as accurately as possible by circling the correct option and providing additional information in the spaces provided.

A. Knowledge of Languages:

1. English is my first language / second language / other

2. I have taken Core French / French Immersion since grade

3. I would say my level of French is: Speaking: basic / good / excellent / fluent Understanding: basic / good / excellent / fluent Reading: basic / good / excellent / fluent Writing: basic / good / excellent / fluent

4. I speak/understand/read/write another language(s): yes no

5. If yes, what language(s)?

6. My level of the other language is:

Speaking:

basic / good / excellent / fluent Understanding: basic / good / excellent / fluent Reading: basic / good / excellent / fluent Writing: basic / good / excellent / fluent

7. My level of Spanish is: Speaking: basic / good / excellent / fluent Understanding: basic / good / excellent / fluent Reading: basic / good / excellent / fluent Writing: basic / good / excellent / fluent

B. Experience with and Attitude toward Languages:

1. I have lived in another country: yes no

2. If yes, a) What country?

b) For weeks / months / years.

3. I have learned another language at school:

4. I have relatives who speak another language:

5. If yes, a) what language?

b) What is the relative's relationship to you?

6. I like languages: yes__ no

7. I would say I'm good with languages: yes __ no ___ not sure

8. Learning languages is: fun/boring/exciting/easy/difficult/interesting/uninteresting/useful/other

C. Learning Style:

1. I learn better when I associate the language with pictures / words / when I hear it / when I can work with it.

2. I would say I am a: visual learner / auditory learner / tactile-kinesthetic learner / other

3. I learn better when I work in small groups / in pairs / on my own.

4. I would say I am an independent learner / a cooperative learner / both.

D. Area(s) of Study/Interests:

1. My Major(s), Minor, Specialization and Emphasis is/are:

2. I am also interested in:

3. I enjoy reading about:

\section{E. Expectations:}

By the end of this course I expect/hope to be able to 
Name

Thank you for completing this questionnaire. I hope that you found this course interesting and useful. The purpose of this questionnaire is to evaluate the method used to teach the classes. Please take some time to answer the questions as accurately as possible by circling the correct option and providing additional information in the spaces provided.

Scale: 1 = strongly disagree, $2=$ disagree, $3=$ not sure, $4=$ agree, $5=$ strongly agree

\section{A. Content: Texts/Questions}

1. The content of the texts was appropriate for my language level.

$1 / 2 / 3 / 4 / 5$

2. The themes and content of the texts were interesting.

3 . The content of the texts was useful.

$1 / 2 / 3 / 4 / 5$

4. The number of texts was appropriate.

$1 / 2 / 3 / 4 / 5$

$1 / 2 / 3 / 4 / 5$

5 . The texts were a good length.

$1 / 2 / 3 / 4 / 5$

6 . The questions were easy to understand.

$1 / 2 / 3 / 4 / 5$

7. The questions helped me to understand the text.

$1 / 2 / 3 / 4 / 5$

Additional comments:

\section{B. Improvement:}

1. I feel that there was improvement in my knowledge of content.

2. I feel that there was improvement in my pronunciation.

3. My level of confidence with the language improved.

4. I feel that I have increased my vocabulary.

5. My comprehension has improved.

Additional comments:

\section{Method:}

1. I felt comfortable using this method.

2. I enjoyed working independently to understand the texts.

3. I enjoyed working cooperatively with my classmates and the instructor.

4. My knowledge of other languages helped me to understand Spanish.

5. I learned when and how to use resources to find information.

6 . I enjoyed reading about people and places in Spanish.

7. I learned about the culture of Spanish-speaking countries.

8. I participated actively in the learning process.

$1 / 2 / 3 / 4 / 5$

9. I learned a lot about the Spanish language.

$1 / 2 / 3 / 4 / 5$

Additional comments:

\section{Knowledge of Spanish:}

1. I would say my level of Spanish is now: Speaking:

\section{Understanding:}

Reading:

Writing: basic / good / excellent / fluent basic / good / excellent / fluent basic / good / excellent / fluent basic / good / excellent / fluent

\section{E. Attitude toward Languages:}

1. I like languages: yes no __ not sure

2. I would say I'm good with languages: yes__ no n__ not sure

3. Learning languages is:

Fun / boring / exciting / easy / difficult / interesting / uninteresting / other

\section{F. Course:}

1. The course was fun / interesting / useful / boring / other

2. I liked the fact that the focus was on reading.

3. The activities of the labs and conversation sessions complemented the seminars.

4. I would prefer to learn more oral Spanish.

5. I feel motivated to continue to learn Spanish.

6. I would like to learn more Spanish using this method.

7. How does this course compare to other language learning experiences you have had? 